\title{
Long term radiographic evaluation of a potentially impacted and dilacerated tooth into complete transposition

\author{
Hassan Ali shafiee ${ }^{1}$, Golnaz Nahvi ${ }^{1}{ }^{*}$, Farzad Aghdashi ${ }^{2}$, Shahryar Karami ${ }^{3}$ \\ ${ }^{1}$ Department of Orthodontics, School of Dentistry, Shahid Beheshti University of Medical Sciences, Tehran, Iran \\ ${ }^{2}$ Department of Oral and Maxillofacial Surgery, School of Dentistry, Shahid Beheshti University of Medical Sciences, Tehran, Iran \\ ${ }^{3}$ Department of Orthodontics, School Of Dentistry, Tehran Medical Sciences, Islamic Azad University, Tehran, Iran
}

\section{Abstract}

Background: Anomalies such as ectopic tooth eruption and transposition are challenging situations that may complicate the orthodontic treatment. Some of these anomalies can only be diagnosed and managed by accurate long-term radiographic examination. Delayed diagnosis could impede simple preventive orthodontic measurements and cause more costly and consuming future complicated orthodontic treatment.

Case Description: We described in detail the long term follow-up of a young female patient referring with a unilateral ectopic eruption. The patient was under close observation and regular orthodontic treatment to guide a unilateral ectopic eruption into a complete transposition of an upper left canine and first premolar. The long term follow-up revealed stables results.

Practical Implications: Early diagnosis and preventive measurements in patients suffering from anomalies such as ectopic eruption is crucial in order to avoid further complications such as tooth impaction and dilaceration formation and adopting the best choice of treatment.
Keywords: Tooth transposition; ectopic eruption; maxillary canine; orthodontic treatment

Citation: Ali Shafiee H, et al. (2021) Long term radiographic evaluation of a potentially impacted and dilacerated tooth into complete transposition Dentistry 3000. 1:a001 doi:10.5195/d3000.2021.138 Received: January 14, 2021

Accepted: April 22, 2021

Published: July 30,2021

Copyright: (C2021 Ali Shafiee H, et al. This is an open access article licensed under a Creative Commons Attribution Work 4.0 United States License. Email: golnaznahvi@gmail.com

\section{Introduction}

Eruption patterns and timing of individual teeth is affected by various items like ethnicity, geographical area, race, hormonal factors, gene pool, gender, social status, growth and nutrition [1]. The canine is the tooth with the highest percentage of problems of space in the dental arch and its ectopic eruption and eruption in a high zone is common [2-3].

Transposition is a kind of ectopic eruption, defined as the positional interchange of two adjacent teeth in the same quadrant of the dental arch [4]. The most common type of transposition is between the canine and first maxillary premolar.

Unilateral transposition has been reported more often than bilateral transposition, with the left side more frequently involved than the right [5-7]. Tooth transposition is a rare anomaly of dentition that interchanges position of two teeth that are adjacent to each other
[8]. The period of transition from primary dentitions to permanent one between the age of 6 and 12 years is often a time of worry for parents [9].

In the population the prevalence of dental transposition that is considered as subdivision of the ectopic eruption is about $0.4 \%$ [10]. Transposition may be complete or incomplete and each of those demands a different management protocol and considerations [11-14]. 
Transposition is often accompanied by other dental anomalies such as dilacerations in the involved teeth [6].

Dilacerations of a tooth is when there is a typical bend in the crown or root [14-17]. This complication make endodontic and surgical procedures difficult to carry out [18]. This malformation of the root is not detected in routine clinical examination and as a result it may be underestimated in many cases [19-22].

Current research shows a high occurrence of dilaceration concurrent with other dental developmental anomalies such as tooth transposition [23]. The nontreatment approach for an ectopically erupting tooth may lead to root resorption, dilaceration of the neighboring teeth, development of cysts, or ankylosis and the need for more complicated and costly orthodontic treatments.

Early diagnosis and careful follow-up of developing dentition is crucial for early intervention and correction of eruptive anomalies. Sometimes guiding a tooth into a transposed location can be beneficial in order to prevent abnormalities such as dilacerations or resorption and remove the need for tooth extraction and future complex orthodontic measurement.

Therefore, in the current study we present a case with ectopic canine eruption followed-up regularly for a long-term period and treated into a complete transposition to prevent canine impaction and dilaceration forming in the adjacent first premolar.

\section{Materials and Methods}

\section{Case presentation}

A healthy seven-year-old patient in the early mixed dentition stage was referred for orthodontic consultation. Her extra-oral examination showed symmetrical craniofacial features with average
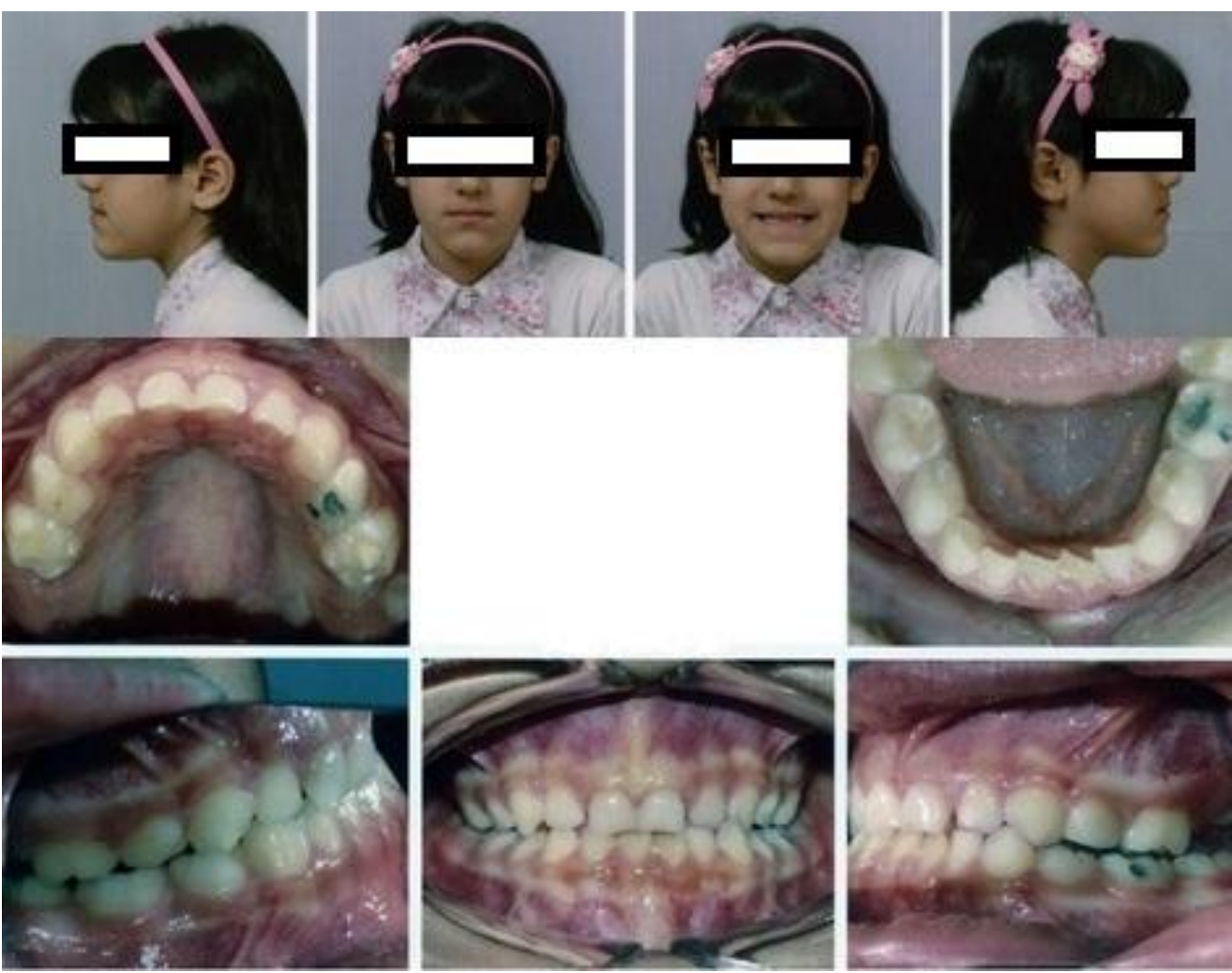

Figure 1. Intra-oral and extra-oral views of the upper and lower dental arch.

facial proportions. Nothing abnormal was detected during a functional evaluation of the stomatognathic system and the temporomandibular joint (TMJ). Intra-oral examination revealed a mesial step malocclusion in primary dentition and an edge-toedge relationship of left upper and lower lateral incisors (Figure 1).

\section{Radiographic evaluations}

The upper left canine showed an ectopic position compared to the contralateral upper right canine (Figure 2). The patient was treated with removable palatal expander with a tong-guard. She was under regular orthodontic observations until the eruption of

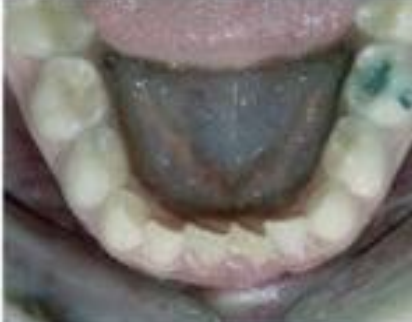




\section{口entistrப $\exists \square \square \square$}

Vol 9 No 1 (2021) DOI 10.5195/d3000.2021.138

the lower incisors.

At this stage stripping of lower deciduous canines was performed to create enough space for lower incisors eruption. At the two-year follow-up, the ectopic position of upper left canine was still obvious with a close proximity to upper left premolar root (Figure 3).

At the three-year and four-year follow-ups, the upper right primary canine had exfoliated, leaving its space vacant. The upper right canine showed a downward movement adjacent to right lateral incisor toward the dental arch but the upper left canine did not follow the regular eruptive path (Figures 4 and 5).

The first and second deciduous molars were extracted with three months interval. At the five-year follow-up at the age of 12 , panoramic and CBCT views of upper dental arch revealed impaction of upper left canine with the canine crown proximity to upper left first premolar palatal root.

Left canine long axis was parallel to the premolars, therefore determining a complete transposition anomaly of the canine. In addition, dilaceration formation in buccal root of left first premolar is clearly evident

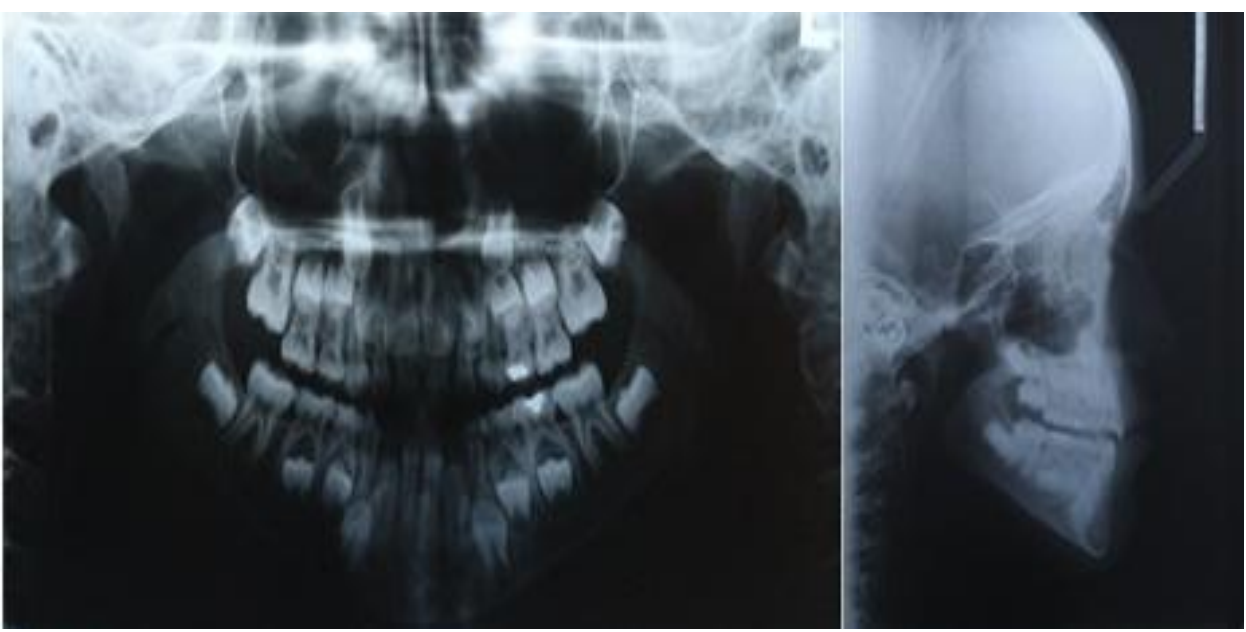

Figure 2. The initial panoramic and cephalogram radiographs of the patient at the age of 7.

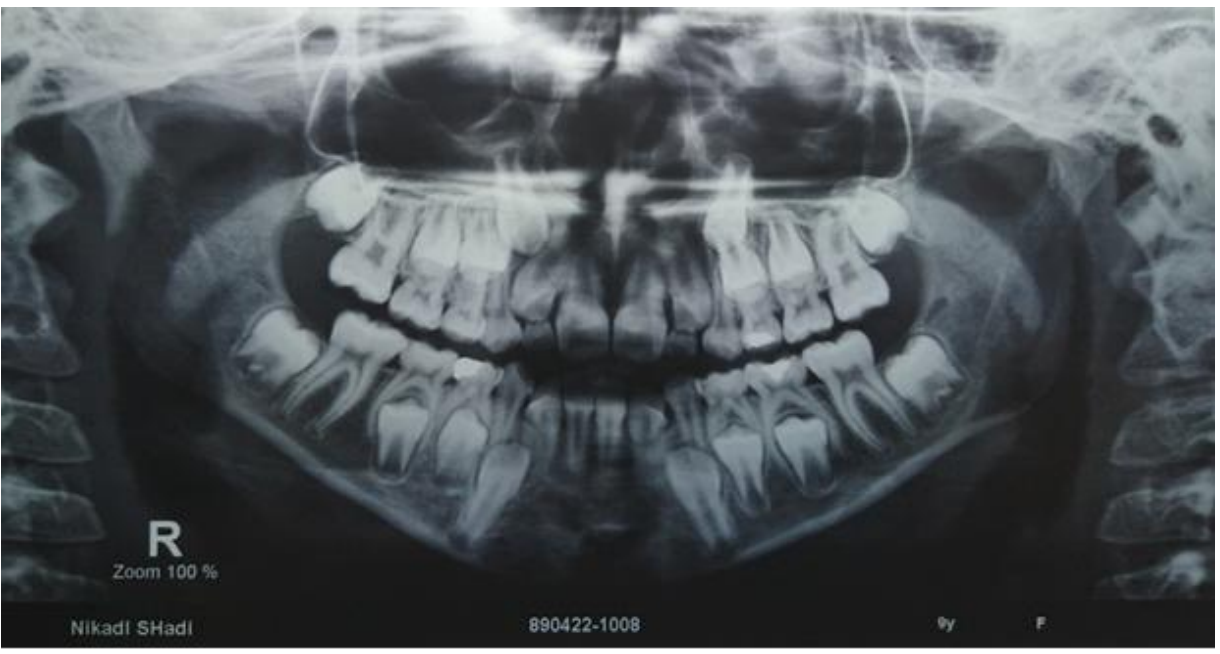

Figure 3. The panoramic radiograph of the patient after two years follow-up at the age of 9.

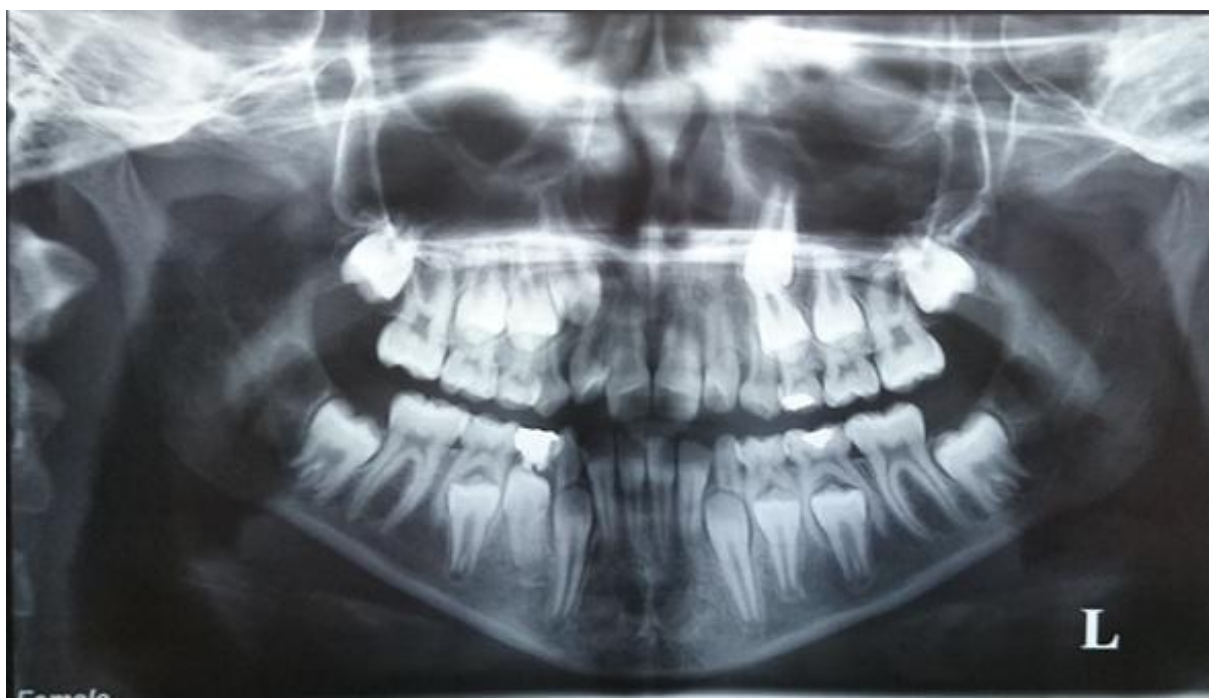

Figure 4. panoramic radiograph of the patient after three years follow-up at the age of 10 . 


\section{口entistrப $\exists \square \square \square$}

Vol 9 No 1 (2021) DOl 10.5195/d3000.2021.138

from different views. The follicular space of upper left canine is 2.97 $\mathrm{mm}$ and is seen in CBCT crosssectional view number 10. All the CBCT sections were prepared in real size with the slice thickness and interval of $1.00 \mathrm{~mm}$ (Figures 6 and 7).

The patient was informed that no intervention at this dental developmental stage might complicate future orthodontic mechanics by increasing the risk of canine impaction and first premolar buccal root dilaceration and resorption. Therefore two different treatment alternatives were discussed for the patient.

\section{Treatment alternatives}

The first treatment option for this case included the extraction of left first premolar to prevent canine impaction, first premolar buccal root dilaceration, resorption and retaining the remaining site for future implant-supported prosthesis in place of extracted first premolar.

The second alternative was to guide the upper left premolar into canine position in order to avoid any extraction and root dilaceration formation and preserving the integrity of upper dental arch. The patient was not willing to extract any permanent

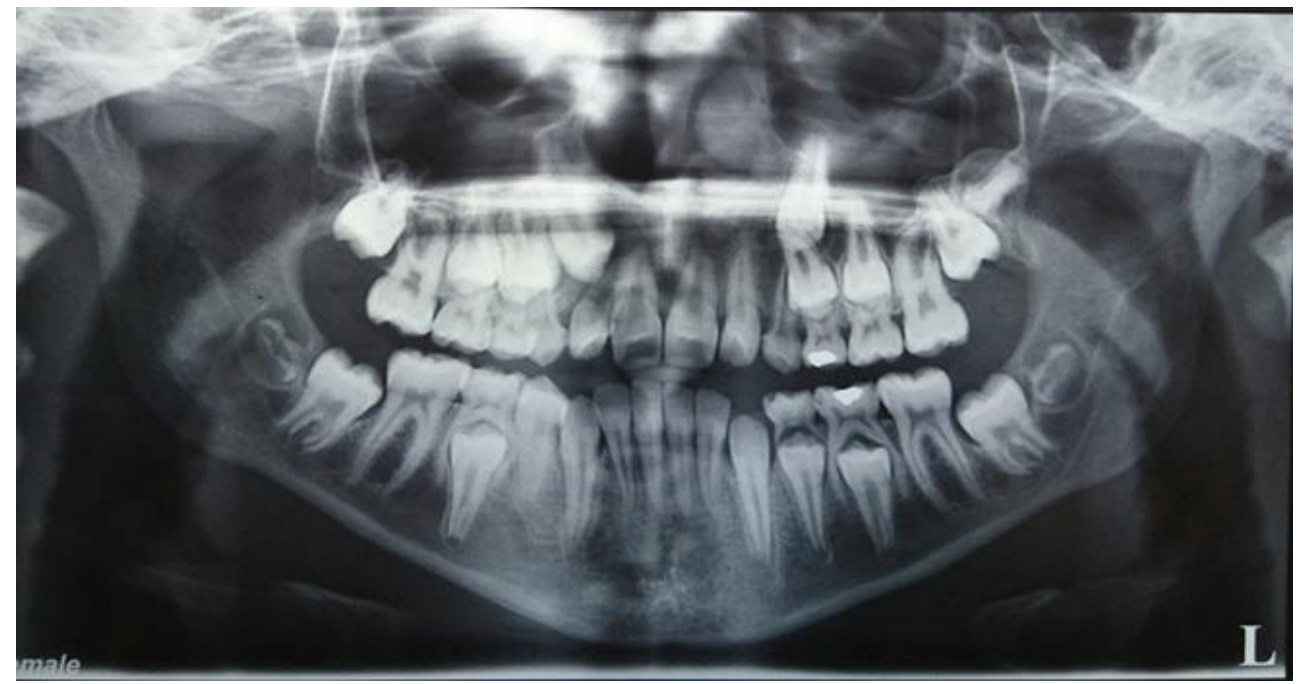

Figure 5. The panoramic radiograph of the patient after four years follow-up at the age of 11.

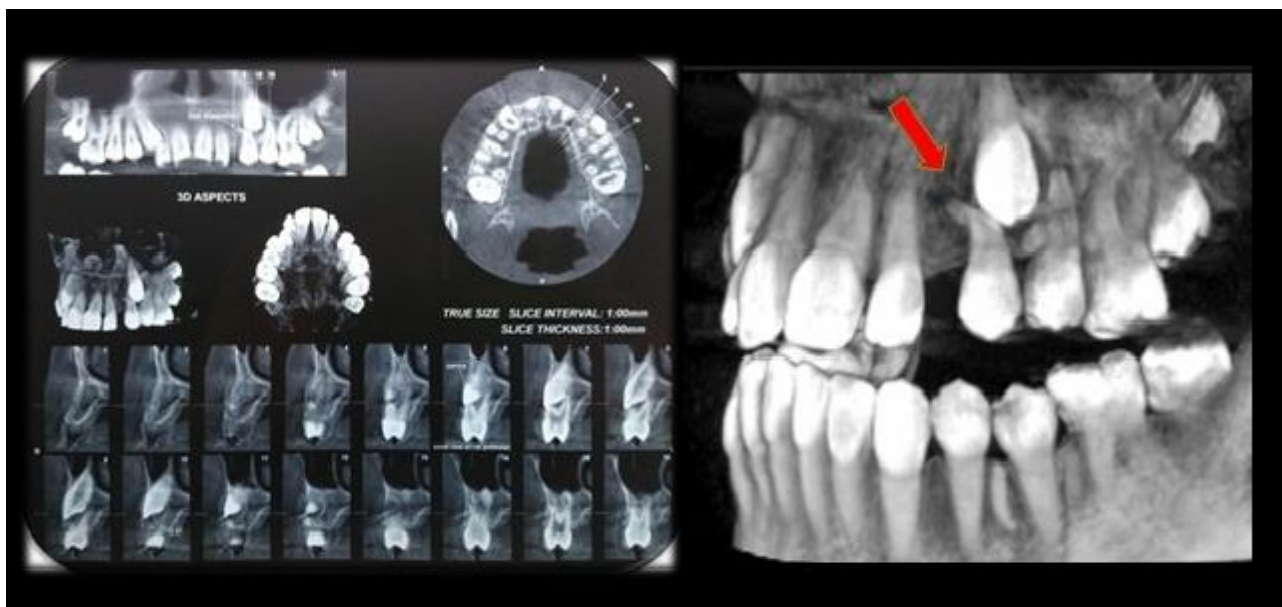

Figure 6. The CBCT views of the patient after five years follow-up at the age of 12.

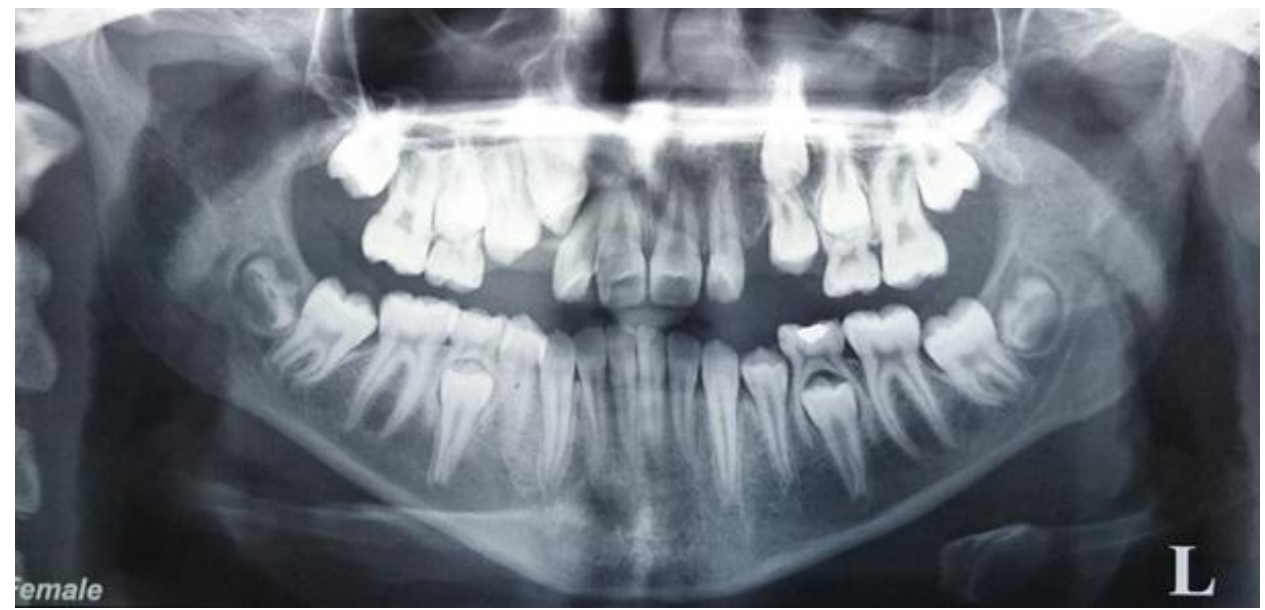

Figure 7. The panoramic view of the patient after five years follow-up at the age of 12. 
entistఁ

tooth so the second treatment option was selected by the patient after thorough discussion of the two options.

\section{Treatment procedure}

Orthodontic treatment was commenced in the upper arch immediately in order to prevent further contact of the canine with premolar buccal root. The primary treatment goal was to reposition the transposed canine into the first premolar site and align the labial segment of both arches. A non-extraction orthodontic treatment was chosen and patient was under rapid maxillary expansion for three months.

Then fixed appliances were used on all upper teeth. The $0.022 x$ 0.025 " MBT prescription (3M Unitek, USA) was placed with 0.014" nickel titanium [24] wire for initial leveling and alignment of the teeth. As treatment progressed, 0.018 " stainless steel wire was placed in the maxillary arch with NiTi open-coil spring between maxillary left canine and first premolar for mesialization of maxillary first premolar and creating space for alignment of the permanent maxillary left canine (Figure 8). When enough space for the canines was

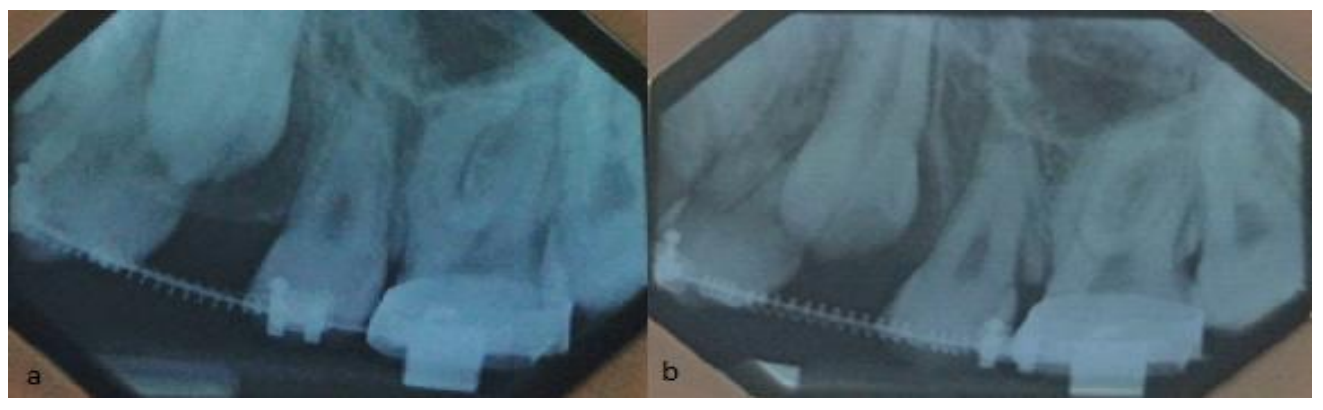

Figure 8. The periapical view of upper left canine during orthodontic alignment at the age of 13 (a) and 14 (b).

obtained, they were bonded and included in the arch wire.

Treatment sessions included regular recalls for appliance adjustment and reinforcement of oral hygiene measures.

Following the transposition of left canine and first premolar, reshaping was also carried out for the maxillary right first premolar to look like canine. The treatment continued to bond the lower arch and establish the final ideal occlusion into class I canine and molar relationships establishment.

\section{Results}

\section{Clinical results}

The patient records showed that all treatment objectives were achieved. The eleven year followup of the patient showed that the case's preventive orthodontic treatment was proven successful.
At first glance, the possibility of orthognathic surgery in the future for treatment of skeletal class III was quite possible, but this treatment option was abandoned with preventive treatments such as an expansion removable plate containing a tang guard at the appropriate age, long-term followup, as well as high patient satisfaction with orthodontic treatment. Also, the patient's intraoral record shows the complete success of preventive treatment with transposition of first premolar instead of canine.

As shown in figure 9, the occlusal view indicates the true transposition of the canine and the first premolar in the left side at the age of 18 years. Ideal overjet and overbite were achieved and also transverse correction was done so that no crossbite was seen after treatment. 


\section{口епடistr $\exists \square \square \square$ \\ Vol 9 No 1 (2021) DOI 10.5195/d3000.2021.138}

\section{Radiographic results}

The panoramic and periapical radiographs showed good parallelism of roots and normal structure of the periodontium, roots, and surrounding tissues. No sign of root resorption or other damage to the teeth were seen in the eleven-year follow-up period.

Also, no evidence of caries was found during the long follow-up period, and the final panoramic radiograph was free of any dental pathology, which showed good hygiene and continuous hygiene training during the treatment period (Figures 9 and 10).

\section{Discussion}

Regular patient follow-up from the early stages of tooth development offers opportunities to intercept and correct some types of developmental abnormalities at the proper time, which might be more burdening in future.

In this case of ectopic canine eruption timely intervention led to satisfactory results by replacing the canine with adjacent premolar to avoid dilaceration formation in buccal root of the left premolar. Correct intercuspation between the upper and lower teeth was achieved, without any aesthetic or functional alterations. Orthodontic treatment was conducted without
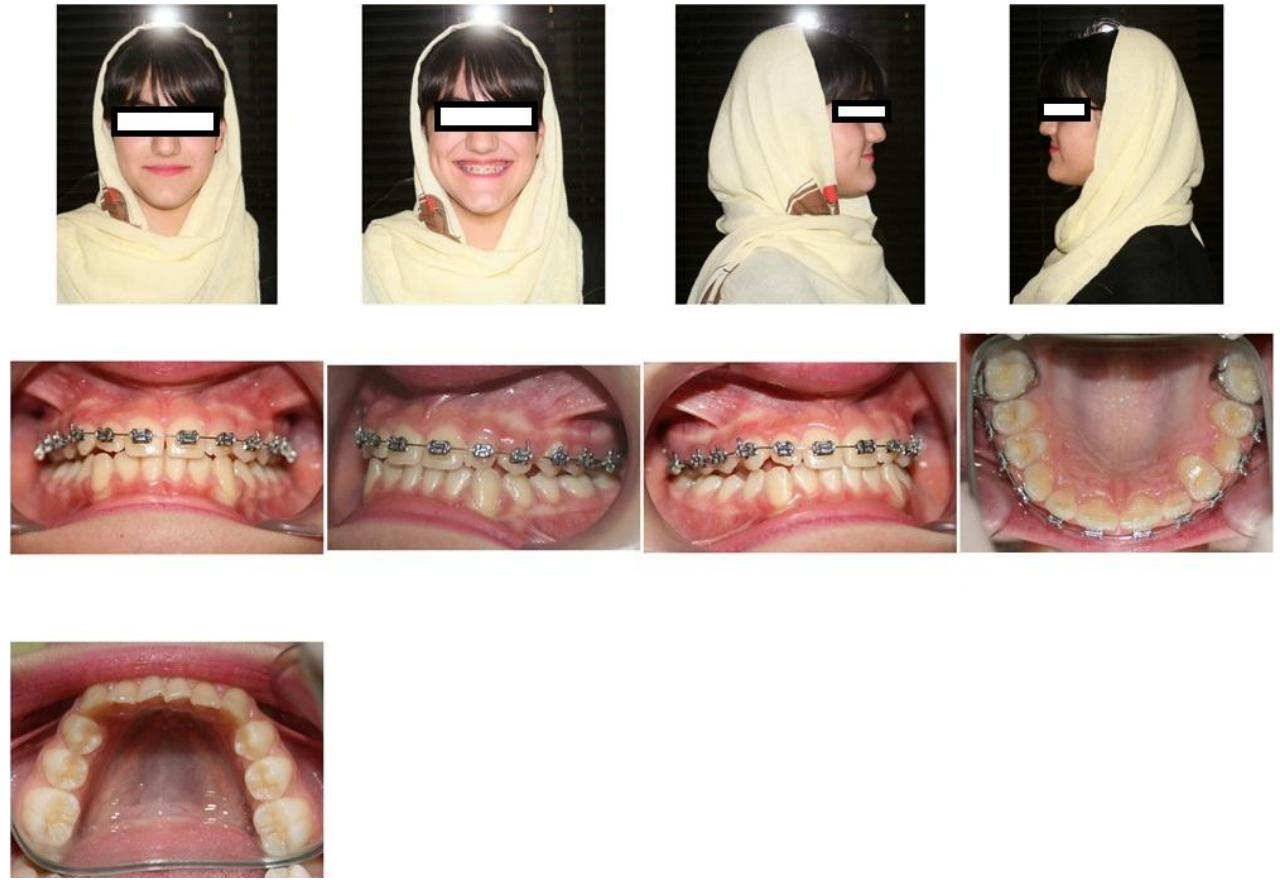

Figure 9. Intra-oral and extra - oral views of the upper and lower dental arch showing successful canine-premolar transposition.

much difficulty or complexity. Side effects that may arise in these situations in case of nointervention protocol, such as: root resorption, dilacerations or impactions were prevented by regular long-term observations.

Previous studies have debated the etiology for variation of root morphology in maxillary first premolar. Even though certain genes such as Msx1 and Wnt have been reported to play a role [2527] most studies indicated that the variation of first premolar roots was related to environmental conditions, particularly the impaction of adjacent canines [28]. The concluded that if canine impaction is not treated it can cause serious harm to adjacent teeth such as root resorption in a large percentage of first premolars [28].

In a recent case report unilateral premolar extraction is conducted in a case with complete transposition of upper canine and first premolar. They recommended that in case of unilateral extraction in transposition cases care must be taken in order to prevent midline shift and development of arch asymmetry during orthodontic intervention [29]. As unilateral extractions in these cases may complicate orthodontic treatment 


\section{Dentistry}

Vol 9 No 1 (2021) DOI 10.5195/d3000.2021.138

choosing a non-extraction protocol to establish a complete transposition could facilitate orthodontic treatment with more predictable mechanics and reasonable results.

Although canine substitution with premolar may be a suitable alternative treatment in some ectopically displaced canines, it needs some certain considerations in order to achieve optimal aesthetic and functional results such as: controlling the canine torque to move the root palatally to hide the root prominence and the premolar torque to move the root buccally to mimic the canine bulge and prevent occlusal interference of the palatal cusp [30].

In early research results it was speculated that root dilacerations of first maxillary premolars were a probable cause for canine impaction [31]. Then, future studies reported that the developing first premolar root may be more susceptible to dilacerations in response to an impacted canine [32-34]. So prevention of canine impaction by transposing it with first premolar is a reasonable treatment approach to prevent dilaceration formation in a first premolar in the stage of root development. Thus proper case selection at the



Figure 10. The panoramic radiograph of the patient after eleven years follow-up at the age of 18

suitable age when the premolar root adjacent to ectopic canine is forming is critical because after complete dilaceration occurs any bodily movement to transpose canine with first premolar can lead to serious side effects such as root resorption.

This method was conducted in the presented patient and led to successful aesthetic and functional results. The key factor for successful management of this case is long term follow-up, excellent patient cooperation and regular attendance of orthodontic treatment sessions.

Root morphology is a fundamental issue in planning orthodontic tooth movements as some root variations such as root dilacerations may cause detrimental side effects such as root resorption during orthodontic tooth movements. Therefore, a thorough knowledge of root anatomy could be helpful in prevention of some iatrogenic side effects. A recent study on Iranian population revealed that the prevalence of root dilacerations is estimated equal between males and females. In addition, the prevalence of dilacerations was estimated $1.65 \%$ over ally different teeth types [35].

Maxillary canine-first premolar transposition is not a common phenomenon. Researches revealed that maxillary canine-first premolar transposition was found to have a lower frequency than the maxillary canine-lateral central transposition [36]. In this case we aimed to intentionally guide a maxillary canine and premolar into a complete transposition so that the potentially first premolar root dilaceration was prevented successfully. 
In a study by Grisar et al. the relationship between threedimensional position of the impacted canine and associated surrounding tissues abnormality was measured. The authors concluded that the most common associated pathologies was dilaceration of the root and resorption of a neighboring tooth [37]. These findings were in line with the current study results which showed evident dilacerations formation in the first premolar adjacent to the impacted canine in the current case.

\section{Conclusion}

Based on the results of this study, we can suggest that: Careful follow-up of the patient at an early age allows the detection of veiled developmental anomalies.

Accurate diagnosis and timely intervention in patients with ectopic eruption and other dental eruptive abnormalities could diminish, prevent or eliminate serious complications such as root dilacerations, resorption or canine impaction. Prompt intervention can facilitate orthodontic treatment and prevent any complex and timely future treatments.

\section{Funding}

There is no financial support.

\section{Acknowledgments}

The authors would like to thank the Department of Orthodontics, School of dentistry, Shahid

Beheshti and Islamic Azad University of Medical Sciences, Tehran, Iran.

\section{Conflicts of Interest}

The authors declare no conflict of interest.

\section{References}

1. Changes in the Sequence of Eruption of Permanent Teeth; Correlation between Chronological and Dental Age and Effects of Body Mass Index of 515-year-old Schoolchildren. Khan AS, Nagar P, Singh P, Bharti M. International journal of clinical pediatric dentistry. 2020;

13(4):368-380.

2. Management of impacted canines. Bishara SE, Kommer DD, McNeil MH, Montagano LN, Oesterle $\amalg$, Youngquist HW. American journal of orthodontics. 1976; 69(4):371-387.

3. Rohani K, Eslamian L, Nahvi G. Correlation between maxillary cuspid impaction with available space and anomalies of maxillary lateral incisors. Dental Research Journal. 2021 Jan 1;18(1):27.

4. Tooth transpositions - a review of the literature and treatment considerations. Shapira Y, Kuftinec MM. The Angle Orthodontist. 1989; 59(4):271-276.

5. Canine transposition as autosomal recessive trait in an inbred kindred. Feichtinger $C$, Rossiwall B, Wunderer $\mathrm{H}$. Journal of dental research. 1977; 56(12):1449-1452.

6. Maxillary tooth transposition: correct or accept? Ciarlantini R, Melsen B. American Journal of Orthodontics and Dentofacial Orthopedics. 2007; 132(3):385394.

7. The presentations and management of transposed teeth. Weeks E, Power S. British dental journal. 1996; 181(11):421.

8. Canine Transposition Prevalence, Distribution and Treatment Considerations among Orthodontic Patients. Finkelstein T, Shapira Y, Pavlidi AM, Davidovitch $\mathrm{M}$, Blumer $\mathrm{S}$, Schonberger S, Shpack N. The Journal of clinical pediatric dentistry. 2020; 44(4):268-273.

9. Sockalingam SNMP, Zakaria ASI, Khan KAM, Azmi FM, Noor NM Simple Orthodontic Correction of Rotated Malpositioned Teeth Using Sectional Wire and $2 \times 4$ Orthodontic Appliances in MixedDentition: A Report of Two Cases. Case reports in dentistry, 2020. 
2020, 6972196 DOI:

https://doi.org/10.1155/2020/697 2196.

10. Orthodontic treatment of unilateral cleft lip and palate associated with maxillary canine/premolar transposition: case report. Souza RMd, Oliveira HTd, Farret MM. Dental press journal of orthodontics. 2020; 25(3):54-64.

11. Orthodontic management of a complete and an incomplete maxillary canine-first premolar transposition. Lorente $\mathrm{C}$, Lorente $P$, Perez-Vela M, Esquinas C, Lorente T. The Angle Orthodontist. 0(0):null.

12. Isolation and screening of extracellular anticancer enzymes from halophilic and halotolerant bacteria from different saline environments in Iran. Zolfaghar $M$, Amoozegar MA, Khajeh K, Babavalian H, Tebyanian H. Mol. Biol. Rep. 2019; 46(3):3275-3286.

13. Histologic analyses of different concentrations of TritonX-100 and Sodium dodecyl sulfate detergent in lung decellularization.

Tebyanian H, Karami A, Motavallian E, Aslani J, Samadikuchaksaraei A, Arjmand B, Nourani MR. Cell Mol Biol (Noisyle-grand). 2017; 63(7):46-51.
14. Current and advanced nanomaterials in dentistry as regeneration agents: an update. Yazdanian M, Rahmani A, Tahmasebi E, Tebyanian $\mathrm{H}$, Yazdanian A, Mosaddad SA. MiniRev. Med. Chem. 2020; 20(1-21.

15. Current biocompatible materials in oral regeneration: a comprehensive overview of composite materials. Tahmasebi E, Alam M, Yazdanian M, Tebyanian H, Yazdanian A, Seifalian A, Mosaddad SA. J Mater Res

Technol. 2020; 9(5):11731-11755.

16. Fabrication and properties of developed collagen/strontiumdoped Bioglass scaffolds for bone tissue engineering. Mosaddad SA, Yazdanian M, Tebyanian $\mathrm{H}$, Tahmasebi E, Yazdanian A, Seifalian A, Tavakolizadeh M. J. Mater. Res. Technol. 2020; 9(6):14799-14817.

17. Current herbal medicine as an alternative treatment in dentistry: In vitro, in vivo and clinical studies. Moghadam ET, Yazdanian M, Tahmasebi E, Tebyanian $\mathrm{H}$, Ranjbar R, Yazdanian A, Seifalian A, Tafazoli A. Eur. J. Pharmacol. 2020; 889(173665.

18. Prevalence of Shape-related Developmental Dental Anomalies in India: A Retrospective Study. Goswami M, Bhardwaj S, Grewal
N. International journal of clinical pediatric dentistry. 2020;

13(4):407-411.

19. Pawlaczyk-Kamieńska T, Winiarska H, Kulczyk T, Cofta S Dental Anomalies in Rare, Genetic Ciliopathic Disorder-A Case Report and Review of Literature. International journal of environmental research and public health, 2020. 17, DOI:

https://doi.org/10.3390/ijerph171 24337.

20. Cell Toxicity and inhibitory effects of Cyperus rotundus extract on Streptococcus mutans, Aggregatibacter actinomycetemcomitans and Candida albicans. Khojaste M, Yazdanian M, Tahmasebi E, Shokri M, Houshmand B, Shahbazi R. Eur J Transl Myol. 2018; 28(4):7917.

21. Fabrication and properties of

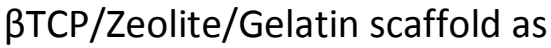
developed scaffold in bone regeneration: in vitro and in vivo studies. Yazdanian M, Tabesh $\mathrm{H}$, Houshmand B, Tebyanian $\mathrm{H}$, Soufdoost RS, Tahmasebi E, Karami A, Ghullame S. Biocybern Biomed Eng. 2020; 40(4):16261637.

22. In vitro and in vivo evaluation of novel Tadalafil/ $\beta$-TCP/Collagen scaffold for bone regeneration: A rabbit critical-size calvarial defect 
study. Soufdoost RS, Yazdanian M, Tahmasebi E, Yazdanian A, Tebyanian $\mathrm{H}$, Karami A, Nourani MR, Panahi Y. Biocybern Biomed Eng. 2019; 39(3):789-796.

23. Dental developmental alterations in patients with dilacerated teeth. LedesmaMontes $C$, Jiménez-Farfán MD, Hernández-Guerrero JC. Medicina oral, patologia oral y cirugia bucal. 2018; 24(1):e8-e11.

24. A Pyk2 inhibitor incorporated into a PEGDA-gelatin hydrogel promotes osteoblast activity and mineral deposition. Posritong $\mathrm{S}$, Chavez RF, Chu T-MG, Bruzzaniti A. Biomedical Materials. 2019; 14(2):025015.

25. Sibling similarities in dental development. Garn SM, Lewis AB, Polacheck D. Journal of dental research. 1960; 39(1):170-175.

26. The current markers of cancer stem cell in oral cancers.

Tahmasebi E, Alikhani M, Yazdanian A, Yazdanian M, Tebyanian H, Seifalian A. Life Sci. 2020; 249(117483.

\section{Surgical Suture Assembled} with Tadalafil/Polycaprolactone Drug-Delivery for Vascular Stimulation Around Wound: Validated in a Preclinical Model. Soufdoost RS, Mosaddad SA, Salari $\mathrm{Y}$, Yazdanian $\mathrm{M}$, Tebyanian $\mathrm{H}$,
Tahmasebi E, Yazdanian A, Karami A, Barkhordari A. Biointerface Res Appl Chem. 2020; 10(5):63176327.

28. Prevalence and risk factors of root resorption of adjacent teeth in maxillary canine impaction, among untreated children and adolescents. Rafflenbeul F, Gros CI, Lefebvre F, Bahi-Gross S, Maizeray R, Bolender Y. European Journal of Orthodontics. 2018.

29. Orthodontic Management of Maxillary Canine-first Premolar Transposition by Unilateral Extraction. Adiwirya MSK, Purwanegara MK. Journal of Dentistry Indonesia. 2019; 26(1):44-50.

30. Maxillary canine-first premolar bilateral transposition in a Class III patient: A case report. Potrubacz MI, Tepedino M, Chimenti C. The Angle

Orthodontist. 2016; 86(3):509519.

31. Displacement of Maxillary Canines: A Twist in the Root. Kerrigan J, Sandy JR. British Journal of Orthodontics. 1995; 22(3):275-278.

32. Maxillary canine displacement; further twists in the tale. Chate R. The European Journal of Orthodontics. 2003; 25(1):43-47.
33. Maxillary canine impaction; a final twist in the tale? Chate R. Journal of orthodontics. 2004; 31(1):13-14.

34. Assessing the Prevalence of Musculoskeletal Disorders and Posture Conditions during Work Time of Dentists in a Dentistry Clinic affiliated to a Military College in Tehran in 2017. Yazdanian M, Moradi K, Sobhani V, Akbari H, Kazemipour M, Shamsoddini A, Tahmasbi E. Journal-Mil-Med. 2018; 20(2):222230.

35. Prevalence and distribution of dilacerations in the permanent dentition of an Iranian population. Kuzekanani M, Sadeghi MT. Eur. j. anat. 2019; 23(4):273-277.

36. Transmigration of an impacted mandibular canine. Zvolanek J. Illinois dental journal. 1986; 55(2):86-87.

37. Three-dimensional position of impacted maxillary canines: Prevalence, associated pathology and introduction to a new classification system. Grisar K, Piccart F, Al-Rimawi AS, Basso I, Politis C, Jacobs R. Clinical and Experimental Dental Research. 2019; 5(1):19-25. 